\title{
Evaluation of the Accuracy of Various Disks and Strips for Rapid Culture-Based Gonococcal Antimicrobial Susceptibility Screening Tests in China
}

\author{
Wen-Qi Xu (D) ${ }^{1,2}$ \\ Jing-Wei Liu (iD) ${ }^{1,2}$ \\ Xiao-Yu Zhu ${ }^{1,2}$ \\ Xiao-Li Zheng ${ }^{1,2}$ \\ Kai Chen ${ }^{1,2}$ \\ Xiang-Sheng Chen $\mathbb{1}^{1,2}$ \\ Yue-Ping Yin (1) ${ }^{1,2}$
}

'STD Reference Laboratory, Institute of Dermatology and Hospital for Skin Diseases, Chinese Academy of Medical Sciences \& Peking Union Medical College, Nanjing, People's Republic of China; ${ }^{2}$ STD Reference Laboratory, National Center for Sexually Transmitted Diseases Control, Chinese Center for Disease Control and Prevention, Nanjing, People's Republic of China
Correspondence: Yue-Ping Yin STD Reference Laboratory, Institute of Dermatology and Hospital for Skin Diseases, Chinese Academy of Medical Sciences \& Peking Union Medical College, No. 12, Jiangwangmiao Street, Nanjing, 210042, People's Republic of China $\mathrm{Tel} / \mathrm{Fax}+862585478024$

Email yinyp@ncstdlc.org
Purpose: Neisseria gonorrhoeae, resistant to the first-line treatment option ceftriaxone, is widespread in China from 2016. Nowadays, diverse reagents of disks and strips for rapid gonococcal antimicrobial susceptibility tests used in clinics are culture-based disks diffusion and gradient strips methods. This study aimed to evaluate the accuracy, quality, and availability of almost all disks and strips acquired in the Chinese market and serve as a reference for clinical selection.

Methods: We tested the performance of 15 commercial disks and 9 commercial gradient strips acquired in China, compared with traditional agar dilution method. The overall performance was evaluated by the categorical agreement. The reagent accuracy of gradient strips was assessed by the essential agreement.

Results: A total of 167 gonococcal isolates were used to evaluate antimicrobial disks from three brands. The overall categorical agreements were $71.7 \%$ to $81.8 \%$ for ceftriaxone, less than $58 \%$ for cefixime, $100 \%$ for spectinomycin, over $98 \%$ for ciprofloxacin, below $70.5 \%$ for penicillin, and $73.3 \%$ to $81.8 \%$ for tetracycline. A total of 81 isolates were tested for different gradient strips. Categorical agreements were over $96 \%$ for ceftriaxone, $86.2 \%$ for azithromycin, $62.3 \%$ to $67.1 \%$ for penicillin, $41.9 \%$ to $67.5 \%$ for tetracycline, and $95 \%$ for ciprofloxacin. Essential agreements were $57.7 \%$ to $87.3 \%$ for ceftriaxone, $70 \%$ for azithromycin, $64.9 \%$ to $68.4 \%$ for penicillin, $51.8 \%$ to $71.2 \%$ for tetracycline, and $91.3 \%$ for ciprofloxacin.

Conclusion: Rapid test reagents of disks and strips based on gonococcal culture have suboptimal performance. Disk diffusion for spectinomycin or ciprofloxacin can be recommended for clinical individualized prescription. The gradient strips are of great value to identify ceftriaxoneresistant gonococcal strains. Furthermore, abundant improvements are required for many reagents to further optimize their accuracy till the fulfillment of molecular detection.

Keywords: Neisseria gonorrhoeae, disk diffusion test, gradient strip test, antimicrobial susceptibility

\section{Introduction}

Gonorrhea, caused by Neisseria gonorrhoeae ( $N$. gonorrhoeae), is one of the most prevalent sexually transmitted infections in the world. If left untreated, it can cause adverse fertility outcomes, and facilitate the transmission of HIV. ${ }^{1} N$. gonorrhoeae has become a public health concern globally due to the antimicrobial resistance. $N$. gonorrhoeae, resistant to the first-line treatment option ceftriaxone, has spread in many countries, including China. ${ }^{2-4}$ In China, gonorrhea was the fourth most commonly reported notifiable communicable disease, with 117,938 cases in $2019 .^{5}$ 
However, the isolates collected for antimicrobial susceptibility testing (AST) by the China Gonorrhoeae Resistance Surveillance Program (GRSP) accounted for less than 1.8\% $(2344 / 133,156)$ in $2018,{ }^{5-7}$ and these tests were totally based on the traditional agar dilution method. Although the agar dilution method is the gold standard method for AST, it is a time- and labor-consuming method and not suitable for rapid clinical tests. Ideally, the China-GRSP should strengthen the monitoring of the AMR trends and inform the adjustment of treatment guidelines, yet an alternative and comparative AST method remains necessary.

ASTs in $N$. gonorrhoeae include qualitative tests (disk diffusion assay and molecular tests) and quantitative tests (agar dilution method and E-test method). The agar dilution method is the gold standard method to detect minimum inhibitory concentrations (MICs) and is recommended mainly for reference laboratories. The MIC of gonococcal isolates was defined as the lowest concentration of the antibiotic that inhibited its growth, with which clinicians can prescribe antibiotics precisely and individually. The disk diffusion test is relatively simple and also well accepted. Yet the observed diameter of inhibition-zone is relatively imprecise and cannot be converted into a concrete MIC value. In addition, a recent study reported that disks made of Oxoid show suboptimal accuracy for ceftriaxone and cefixime in clinics compared with values determined by the agar dilution method. ${ }^{8}$ E-test (bioMerieux, France), one of the labeled gradient strip methods, is suitable as an alternative test to the agar dilution method, although it has not been approved by the China Food and Drug Administration. Moreover, the molecular tests have a restricted detection range for predicting specific antimicrobial resistance in gonococcal isolates, such as cephalosporin, ciprofloxacin, and azithromycin resistance. ${ }^{9,10}$ Nonetheless, disk diffusion and gradient strip methods are nowadays the first choice in microbiological laboratories and hospitals world-wide. Improvements of these two methods benefit clinics for their timely and precise prescription.

In this study, we tested the performance of different commercial disks (Oxoid, Binhe, Kangtai) and gradient strips (Oxoid, Antu, Kangtai) acquired in China. By comparing the performance with the traditional agar dilution method for the evaluation of suitability and reliability, we recommend which reagents for clinical use in some resource-limited areas where the agar dilution method cannot be used, based on the price, operational complexity, and result reliability. In the meantime, it bridges the gap before the realization of molecular detection.

\section{Materials and Methods}

\section{Ethics Approval and Consent to Participate}

The gonococcal isolates were collected through the China Gonococcal Resistance Surveillance Programme (ChinaGRSP), and had been saved for use in the present study. The ethics approval for the study was obtained from the Medical Ethics Committee at the Institute of Dermatology, Chinese Academy of Medical Sciences \& Peking Union Medical College (2014-LS-026). Participants no less than 18 years of age who signed an informed consent to provide urine and vaginal and rectal swabs were enrolled in the study. Samples were inoculated, identified, preserved, and transferred as previously described. ${ }^{7}$

\section{Bacterial Strains}

A total of 167 clinical samples from urethral swabs were collected and subsequently cultured on selective ThayerMartin (TM) media. Microscopy, rapid oxidase reaction, and carbohydrate utilization test were used for verification. All gonococcal isolates were preserved in skimmed milk and stored at $-70^{\circ} \mathrm{C}$ as part of routine diagnostics. Strains for further tests were randomly selected, then cultured onto selective TM media and sub-cultured on GC agar base media (Oxide, Hampshire, England) supplemented with 10\% defibrinated sheep blood (Bianzhen Biotechnology, Nanjing, China) and 1\% Iso VitaleX Enrichment (BD Diagnostics, New Jersey, USA) at $36^{\circ} \mathrm{C}$ in a moist $5 \% \mathrm{CO}_{2}$-enrichment atmosphere for 18 to 20 hours.

\section{Agar Dilution Test}

The MICs were determined according to the agar dilution method recommended by WHO as previously described. ${ }^{7}$ The 2016 WHO N. gonorrhoeae reference strains panels $(\mathrm{G}, \mathrm{K}, \mathrm{L}$, and $\mathrm{P}$ ) were included as outer quality control in every batch of experiments. ${ }^{7}$

\section{Disk Diffusion and Gradient Strip Test}

Commercialized disks (Oxoid for ceftriaxone, cefixime, spectinomycin, penicillin, tetracycline, and ciprofloxacin; Binhe for ceftriaxone, cefixime, spectinomycin, penicillin, tetracycline, and ciprofloxacin; Kangtai for ceftriaxone, cefixime, spectinomycin, penicillin, tetracycline, and ciprofloxacin) and commercialized MIC gradient strips (Oxoid for penicillin, tetracycline, and ciprofloxacin; Antu for ceftriaxone, azithromycin, and penicillin; Kangtai for ceftriaxone, penicillin, and tetracycline) were evaluated for their 
antimicrobial susceptibility accuracy. Three manufacturers' antimicrobial disks were assessed for ceftriaxone, azithromycin, cefixime, spectinomycin, penicillin, tetracycline, and ciprofloxacin, in addition to disks from Oxoid for azithromycin. All disks and strips were purchased for routine diagnostic use, and none of the companies had any influence on the design or performance of our study.

All tests were performed in accordance with the manufacturer's instruction and WHO recommendations. ${ }^{11}$ The same GC agar base media supplemented with sheep blood and enrichment were used for strain culture. The identical reference strains used in agar dilution test were included in every batch of testing. The results were interpreted by reading the diameter of the inhibition zone measured with a ruler (disk diffusion) or the lowest growth inhibition concentration (gradient strip). Nearest diameter breakpoints and MIC breakpoints from the Clinical and Laboratory Standards Institute (CLSI; em100.edaptivedocs.net/dashboard.aspx) were used for the categorization of susceptibility (S), intermediate (I), and resistance (R). For azithromycin, where CLSI states no SIR breakpoints, MIC breakpoints from the European Committee on Antimicrobial Susceptibility Testing (EUCAST; www.eucast.org/clinical breakpoints/) were applied.

\section{Statistical Analyses}

The overall performance of the assays was evaluated by determining the categorical agreement (CA). Results were considered CA when isolates had the same susceptible, intermediate, susceptible dose-dependent, and resistant category as the agar dilution method category result. Very major errors were R strains being misclassified as S. Major errors were $\mathrm{S}$ strains being misclassified as R. Minor errors occurred when one method categorized an isolate as I while the other method defined it as S or R, as previously described. ${ }^{12}$ With no breakpoints for azithromycin, we determined the Pearson's correlation tests between diameters and MIC values.

The reagent accuracy of gradient strips was assessed by calculating the essential agreement (EA), which was defined as the percentage of strains with predicted MICs that did not deviate by more than \pm 1 doubling dilution from agar dilution MICs. Ideally, an EA between different tests should be $>90 \%$.

\section{Results}

During each batch of the tests, MIC values obtained from the reference strains were identical or within 1 doubling dilution of those previously reported. ${ }^{13}$
A total of 167 randomly selected gonococcal isolates were used to evaluate antimicrobial disks from three brands. Specific numbers of categorical accuracy are shown in Table 1. For ceftriaxone and cefixime, breakpoints from the CLSI can only distinguish susceptibility and non-susceptibility, with no very major errors or major errors. The overall categorical agreements of disk diffusion method to ceftriaxone were $71.7 \%$ for Oxoid, $73.2 \%$ for Binhe, and $81.8 \%$ for Kangtai, respectively. Categorical agreements for cefixime were suboptimal, showing less than $58 \%$ for all three manufacturers. Categorical agreements for spectinomycin were 100\% because no resistant strains were tested in our study. Likewise for ciprofloxacin, categorical agreements of all three disks surpassed $98 \%$. Concordance rates for penicillin went below to $70.5 \%$. For tetracycline, agreements were 73.3\% (Oxoid), 76.4\% (Binhe), and 81.8\% (Kangtai). Without recommended diameter breakpoints being proposed in CLSI or EUCAST, we calculated the Pearson's correlation coefficients between their diameters and MIC values. Disks for azithromycin made in Kangtai $(r, 0.59)$ correlated better than those from Binhe $(r, 0.52)$ with a statistical significance.

Nearly 81 isolates were tested for susceptibility accuracy of different gradient strips. The overall performances for five antimicrobials are provided in Table 2. For ceftriaxone, essential agreements were $87.3 \%$ for Antu, and $57.7 \%$ for Kangtai, with categorical agreements for both exceeding $96 \%$. For azithromycin, only strips made from Antu were detected, with $70.0 \%$ and $86.3 \%$, respectively, for its essential and categorical concordance. Gradient strips of Oxiod, Antu, and Kangtai were evaluated for penicillin, with no major or very major errors found, and the agreements ranged from $62.3 \%$ to $68.4 \%$. For tetracycline, accuracy of Oxoid was higher than that of Kangtai. Only strips of Oxoid were purchased for ciprofloxacin, with $91.3 \%$ and $95 \%$ separately for essential and categorical agreements, respectively.

\section{Discussion}

Monitoring the trend of clinical $N$. gonorrhoeae with reduced susceptibility to different antimicrobials is an important task for public health agencies. Yet, currently there is no large-scale comparative study on definite accuracy of different AST agents in China for clinically widely used disks or strips methods. In this study, we evaluated the performance of 27 commercial reagents for disk diffusion and gradient strip methods, using strains randomly 


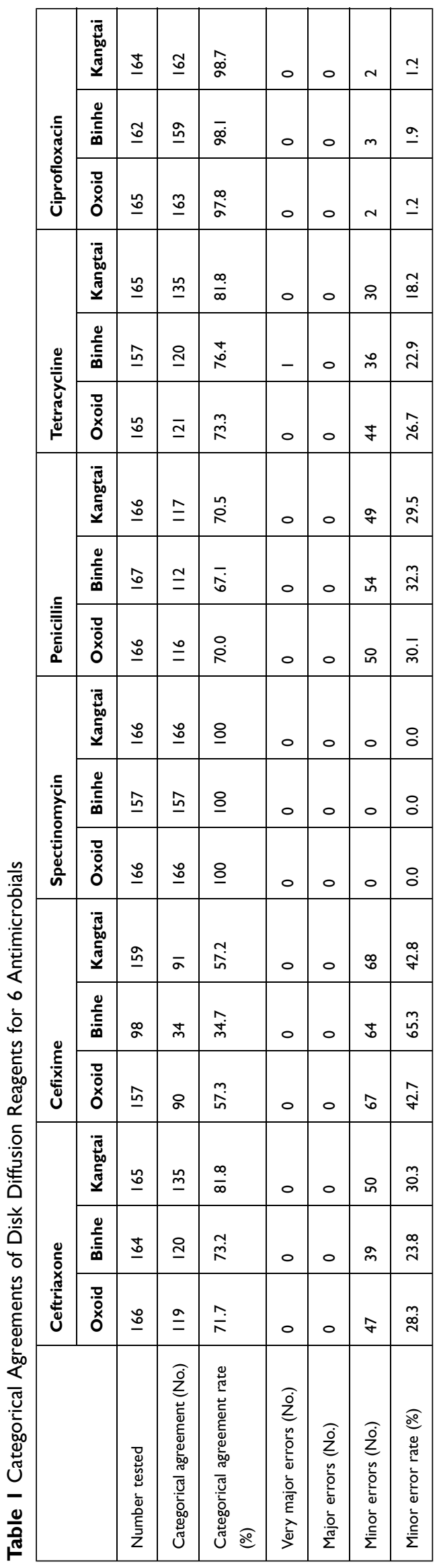

selected from China GRSP, in order to formulate our recommended methods and reagents.

The disk diffusion method is less labor-intensive and easier to carry out than the traditional agar dilution method, which is, rather, suitable for clinical tests to guide individually treatment or find resistant strains for microbial infections. In our comparative study, categorical agreements showed suboptimal results despite being carried out in one laboratory. For ceftriaxone and cefixime, performance rates of six manufacturers were less than accepted percentages (defined as $>90 \%$ for categorical agreements and $<7 \%$ for minor errors), and the performance is worse for cefixime. Agreements in our study, resembling the results conducted in China GRSP, ${ }^{6}$ were much lower than other comparative researches (Oxoid) in foreign countries, all of which showed concordant rates over $89 \% .{ }^{14-16}$ The range of susceptibility values of these two antimicrobials was larger and comprehensive, which may lead to suboptimal agreements in studies conducted in China. All the disks had excellent agreements for ciprofloxacin and spectinomycin, notwithstanding that the clinical strains collected were mostly resistant or susceptible to them, indicating that these two disks may have clinical efficiencies for individual prescription like molecular assay kits, which performed well in the detection of AMR determinants for predicting phenotypic ciprofloxacin and azithromycin resistance. ${ }^{17}$ Disk diffusion has inferior accuracy for tetracycline and penicillin, but reagents from Kangtai performed best. Overall, as cheap and easy as the disk diffusion method is, training and experience are required for reading the results. Reading the diameters of different disks can be subjective, which can affect the outcome, especially for the antimicrobials with low MIC values. Similarly, the E-test method, one of the gradient strip methods, is another alternative AST method. The E-test method has results comparable with the standardized agar dilution method, but is less labor-intensive. However, the E-test strips made by bioMerieux are not commercially available in China, with no allowance of passports and no clinical ratification from CFDA. Strips sold in China perform with the same principle as E-test plastic strips, but are expensive (over \$4.50) due to patent protection. Hence, gradient strips for ceftriaxone and azithromycin in our study are both fabricated domestically. Despite its high concordance in ciprofloxacin, the price made it suboptimal than ciprofloxacin disks. Compared with overseas study on variant gonococcal gradient strips, all the strips tested in our research showed acceptable agreements for ceftriaxone categorical potency. ${ }^{18}$ Strips made by 
Table 2 Categorical and Essential Agreements of Gradient Strip Reagents for 5 Antimicrobials

\begin{tabular}{|l|l|l|l|l|l|l|l|l|l|}
\hline & \multicolumn{2}{|l|}{ Ceftriaxone } & \multicolumn{2}{l|}{ Azithromycin } & \multicolumn{2}{l|}{ Penicillin } & \multicolumn{2}{l|}{ Tetracycline } & \multicolumn{2}{l|}{ Ciprofloxacin } \\
\cline { 2 - 11 } & Antu & Kangtai & Antu & Oxoid & Antu & Kangtai & Oxoid & Kangtai & Oxoid \\
\hline Number tested & 79 & 78 & 80 & 77 & 77 & 76 & 80 & 81 & 80 \\
\hline Essential agreement (No.) & 69 & 45 & 56 & 50 & 48 & 52 & 57 & 42 & 73 \\
\hline Essential agreement rate (\%) & 87.3 & 57.7 & 70.0 & 64.9 & 62.3 & 68.4 & 71.3 & 51.9 & 91.3 \\
\hline Categorical agreement (No.) & 77 & 75 & 69 & 48 & 50 & 51 & 54 & 34 & 76 \\
\hline Categorical agreement rate (\%) & 97.5 & 96.2 & 86.3 & 62.3 & 64.9 & 67.1 & 67.5 & 42.0 & 95.0 \\
\hline Very major errors (No.) & 0 & 0 & 1 & 0 & 0 & 0 & 3 & 3 & 0 \\
\hline Major errors (No.) & 0 & 0 & 0 & 0 & 0 & 0 & 0 & 0 & 0 \\
\hline Minor errors (No.) & 2 & 3 & 10 & 29 & 27 & 25 & 23 & 44 & 4 \\
\hline Minor error rate (\%) & 2.5 & 3.8 & 12.5 & 37.7 & 35.1 & 32.9 & 28.8 & 54.3 & 5.0 \\
\hline
\end{tabular}

Antu got higher categorical agreements than those by Liofilchem and HiMedia, ${ }^{18}$ but all the rates were below $86.3 \%$. In short, the gradient strips can be recommended as an alternative AST method in order to quickly find out the strains resistant to the first-line option ceftriaxone and control the infection at an early stage, regardless of the brands.

Considering variant kinds of antibiotics, the susceptibility profiles to first-line drugs (ceftriaxone or spectinomycin) can directly be distinguished by rapid tests. But, for other treatment options except ciprofloxacin, qualities of the antibiotic disks and strips, no matter their brands, need to be further improved.

This is the first study comparing so many disks and gradient strips for gonococcal ASTs in the Chinese market. Nevertheless, there are also limitations in our study. First, the time for gonococcal incubation needs to be shortened by changing to a more nutrient-rich medium, or a more suitable incubation environment. In China, most doctors prescribe antibiotics for gonococcal infection just after a smear microscopy instead of waiting days for the AST results. Second, the reagents compared in our study cannot be fully matched in the number of tests because some items were out of stock during the experiment.

In conclusion, the overall performances of antimicrobial disks and strips are suboptimal to the agar dilution method for susceptibility testing in $N$. gonorrhoeae, but some reagents can be recommended for rapid clinical detection and routine antimicrobial resistance surveillance. In detail, disk diffusion for spectinomycin or ciprofloxacin can be recommended as an initial detection for individualized prescription, and the gradient strips are of great value to identify ceftriaxone-resistant gonococcal strains. Furthermore, the price of strips needs to be regulated, and a strict quality assurance (both at manufacturing and reference laboratory level) is required, including internal and external quality controls. Ultimately, before the arrival of a rapid PCR-based molecular method, the traditional disks and strips methods will still dominate the market and are in need of further improvements. Last but not least, all the results were detected from our laboratory, and are intended to serve as primary references for clinical usage.

\section{Acknowledgments}

This work was supported by the National Science and Technology Major Project (2018ZX101010 01-004-003), the Nanjing Incubation Program for National Clinical Research Center (2019060001), and Jiangsu Province Youth Fund Project (BK20180156).

\section{Disclosure}

The authors report no conflicts of interest in this work.

\section{References}

1. Cohen MS, Hoffman IF, Royce RA, et al. Reduction of concentration of HIV-1 in semen after treatment of urethritis: implications for prevention of sexual transmission of HIV-1. Lancet. 1996;349 (9069):1868-1873. doi:10.1016/S0140-6736(97)02190-9

2. Liao M, Bell K, Gu W-M, et al. Clusters of circulating Neisseria gonorrhoeae strains and association with antimicrobial resistance in Shanghai. J Antimicrob Chemother. 2008;61(3):478-487. doi:10.1093/ $\mathrm{jac} / \mathrm{dkm} 544$ 
3. Unemo M, Shafer WM. Antimicrobial resistance in Neisseria gonorrhoeae in the 21st century: past, evolution, and future. Clin Microbiol Rev. 2014;27(3):587-613. doi:10.1128/CMR.00010-14

4. Chen S, Han Y, Yuan L, et al. Identification of Internationally Disseminated Ceftriaxone-Resistant Neisseria gonorrhoeae Strain FC428, China. Emerg Infect Dis. 2019;25(7):1427-1429. doi:10.3201/eid2507.190172

5. PRC N H C. Overview of the national epidemic of infectious diseases in 2019 [EB/OL]. Available from: http://www.nhc.gov.cn/jkj/s3578/ 202004/b1519e1bc1a944fc8ec176db600f68d1.shtml. Accessed November 23, 2021.

6. Wang Q, Zhang R, Liu Q, et al. National guidelines on diagnosis and treatment of gonorrhea in China (2020). Int J Dermatol Venereol. 2020;3(3):129-134. doi:10.1097/JD9.0000000000000072

7. Yin Y, Han Y, Dai X, et al. Susceptibility of Neisseria gonorrhoeae to azithromycin and ceftriaxone in China: a retrospective study of national surveillance data from 2013 to 2016. PLoS Med. 2018;15 (2):e1002499. doi:10.1371/journal.pmed.1002499

8. Han Y, Yin Y, Xu W, et al. Disk-Diffusion Testing Is an Inappropriate Screening Tool for Cephalosporin-Resistant Gonorrhoea Strains in Clinical Practice in China. Infect Drug Resist. 2020;13:2417-2423. doi:10.2147/IDR.S248030

9. Zhou Q, Liu J, Chen S, et al. The Accuracy of Molecular Detection Targeting the Mutation C2611T for Detecting Moderate-Level Azithromycin Resistance in Neisseria gonorrhoeae: a Systematic Review and Meta-Analysis. Antibiotics. 2021;10(9):1027. doi:10.3390/antibiotics 10091027

10. Peterson SW, Martin I, Demczuk W, et al. Multiplex real-time PCR assays for the prediction of cephalosporin, ciprofloxacin and azithromycin antimicrobial susceptibility of positive Neisseria gonorrhoeae nucleic acid amplification test samples. J Antimicrob Chemother. 2020;75(12):3485-3490. doi:10.1093/jac/dkaa360

11. World Health Organization. Manual for the Laboratory Identification and Antimicrobial Susceptibility Testing of Bacterial Pathogens of Public Health Concern in the Developing World. Geneva: World Health Organization; 2003.
12. Chen SC, Liu JW, Wu XZ, et al. Comparison of Microdilution Method with Agar Dilution Method for Antibiotic Susceptibility Test of Neisseria gonorrhoeae. Infect Drug Resist. 2020;13:1775-1780. doi:10.2147/IDR.S253811

13. Unemo M, Golparian D, Sanchez-Buso L, et al. The novel 2016 WHO Neisseria gonorrhoeae reference strains for global quality assurance of laboratory investigations: phenotypic, genetic and reference genome characterization. J Antimicrob Chemother. 2016;71 (11):3096-3108. doi:10.1093/jac/dkw288

14. Mal PB, Jabeen K, Farooqi J, et al. Antimicrobial susceptibility testing of Neisseria gonorrhoeae isolates in Pakistan by Etest compared to Calibrated Dichotomous Sensitivity and Clinical Laboratory Standards Institute disc diffusion techniques. BMC Microbiol. 2016;16(1):236. doi:10.1186/s12866-016-0707-6

15. Liu H, Taylor TJ, Pettus K, et al. Comparing the disk-diffusion and agar dilution tests for Neisseria gonorrhoeae antimicrobial susceptibility testing. Antimicrob Resist Infect Control. 2016;5:46. doi:10.1186/s13756-016-0148-x

16. Singh V, Bala M, Kakran M, et al. Comparative assessment of CDS, CLSI disc diffusion and Etest techniques for antimicrobial susceptibility testing of Neisseria gonorrhoeae: a 6-year study. BMJ Open. 2012;2(4):e000969. doi:10.1136/bmjopen-2012-000969

17. Hadad R, Cole MJ, Ebeyan S, et al. Evaluation of the SpeeDx ResistancePlus $^{\circledR}$ GC and SpeeDx GC 23S 2611 (beta) molecular assays for prediction of antimicrobial resistance/susceptibility to ciprofloxacin and azithromycin in Neisseria gonorrhoeae. $J$ Antimicrob Chemother. 2020;76:84.

18. Jönsson A, Jacobsson S, Foerster S, et al. Performance characteristics of newer MIC gradient strip tests compared with the Etest for antimicrobial susceptibility testing of Neisseria gonorrhoeae. Apmis. 2018;126(10):822-827. doi:10.1111/apm.12887
Infection and Drug Resistance

\section{Publish your work in this journal}

Infection and Drug Resistance is an international, peer-reviewed openaccess journal that focuses on the optimal treatment of infection (bacterial, fungal and viral) and the development and institution of preventive strategies to minimize the development and spread of resistance. The journal is specifically concerned with the epidemiology of antibiotic resistance and the mechanisms of resistance development and diffusion in both hospitals and the community. The manuscript management system is completely online and includes a very quick and fair peerreview system, which is all easy to use. Visit http://www.dovepress.com/ testimonials.php to read real quotes from published authors. 